\title{
Elastic electron scattering by ethyl vinyl ether
}

\author{
M. A. Khakoo and L. Hong \\ Department of Physics, California State University, Fullerton, California 92834, USA
}

B. Kim

Troy High School, 2200 Dorothy Lane, Fullerton, California 92831, USA

\author{
C. Winstead and V. McKoy \\ A. A. Noyes Laboratory of Chemical Physics, California Institute of Technology, Pasadena, California 91125, USA
}

(Received 10 December 2009; published 24 February 2010)

\begin{abstract}
We report measured and calculated results for elastic scattering of low-energy electrons by ethyl vinyl ether (ethoxyethene), a prototype system for studying indirect dissociative attachment processes that may play a role in DNA damage. The integral cross section displays the expected $\pi^{*}$ shape resonance. The agreement between the calculated and measured cross sections is generally good.
\end{abstract}

DOI: 10.1103/PhysRevA.81.022720

PACS number(s): $34.80 . \mathrm{Bm}, 34.80 . \mathrm{Gs}$

\section{INTRODUCTION}

In molecular gases and plasmas, much of the electrondriven chemistry below the first ionization threshold involves dissociative attachment (DA). However, direct collisions involving electrons with kinetic energies of more than a few tens of milli-electron-volts (meV) occur so quickly on the time scale of nuclear motion that dissociative attachment cross sections are usually negligible, except at energies where long-lived electron-molecule complexes (temporary anions, scattering resonances) are formed. Experimental study of DA processes is a highly developed field and typically involves taking negative-ion mass spectra as a function of the incident electron's kinetic energy in order to locate and characterize the resonances as well as identify their dissociation products. Studies of different isotopomers can yield additional information about which bonds are broken (e.g., Refs. [1,2]) while in some recent work, energy- and angle-resolved detection of the anionic fragments provides further insight into the DA process $[3,4]$. However, DA to polyatomics frequently produces rearranged fragment ions of unclear origin, and in any case, even knowing the precise site of bond cleavage may leave the mechanism doubtful. For example, does the electron attach directly to the cleavage site, or does it attach elsewhere and then undergo intramolecular electron transfer? Many examples of each mechanism are known. Recently a controversy has arisen over which mechanism is principally responsible for DA to formic acid, $\mathrm{H}_{2} \mathrm{CO}_{2}$, to produce an $\mathrm{H}$ atom and a formate $\left(\mathrm{HCO}_{2}^{-}\right)$ion. Originally an indirect mechanism, in which the electron is captured by the $\mathrm{C}-\mathrm{O} \pi^{*}$ orbital, was proposed [5], but subsequently it was argued [6] that direct capture into a $\mathrm{C}-\mathrm{H} \sigma^{*}$ orbital was more likely, and the issue remains unresolved $[7,8]$.

Computational studies of DA offer the appealing prospect of resolving such ambiguities by following the evolution of the nuclear wave function on well-characterized electronic surfaces, taking into account nonadiabatic effects as necessary. The relevant theory is well established [9-11]; the challenge, of course, lies in obtaining and characterizing the potential surfaces. Obtaining accurate electronic surfaces for polyatomic molecules, with a well-balanced description of both the neutral molecule and the anion, is already a significant computational challenge, but in the case of dissociative attachment, the anion surfaces must also describe temporary anions-that is, states embedded in the electronic continuum-to which conventional bound-state electronic-structure methods do not apply. Such states must instead be treated by scattering methods that can deal well with the demands of the low-energy electron-molecule collision problem: in particular, low or no symmetry, and large effects due to electron exchange and target polarization.

Because DA tends overwhelmingly to be driven by the shape and Feshbach resonances that decay into the electronic ground state, studying the elastic-scattering cross section itself is a useful first step in elucidating DA mechanisms. Here we report low-energy elastic cross sections for electron scattering by the ethyl vinyl ether molecule, also known as vinyl ethyl ether or ethoxyethene and hereafter referred to as EVE. The structure of EVE is shown schematically in Fig. 1. With its $\mathrm{C}-\mathrm{C}$ double bond adjacent to two $\mathrm{C}-\mathrm{O}$ bonds, $\mathrm{EVE}$ is a test case for studying possible indirect cleavage of $\mathrm{C}-\mathrm{O}$ bonds following initial attachment in a neighboring $\mathrm{C}-\mathrm{C} \pi^{*}$ orbital. Besides the obvious similarity to the controversial case of formic acid mentioned above, such a process is relevant to low-energy electron damage to nucleic acids, for which one proposed mechanism is attachment to the $\pi$ system of a nucleobase followed by intramolecular electron transfer to the backbone and rupture of an esteric bond connecting the phosphate and sugar groups [12-15].

To our knowledge, the only prior study of low-energy electron interactions with EVE is the work of Bulliard et al. [16], who investigated DA to EVE and related compounds. For EVE, they observed the vinylidene $\left(\mathrm{C}_{2} \mathrm{H}_{2}^{-}\right)$, ethanolate $\left(\mathrm{C}_{2} \mathrm{H}_{5} \mathrm{O}^{-}\right)$, and ethenolate $\left(\mathrm{C}_{2} \mathrm{H}_{3} \mathrm{O}^{-}\right)$ions, with a peak in the production of all three ions near $3 \mathrm{eV}$ incident electron energy. The latter two ions can be produced by cleavage of one $\mathrm{C}-\mathrm{O}$ bond or the other, though Bulliard et al. note evidence that some ethenolate is produced by a different mechanism. 


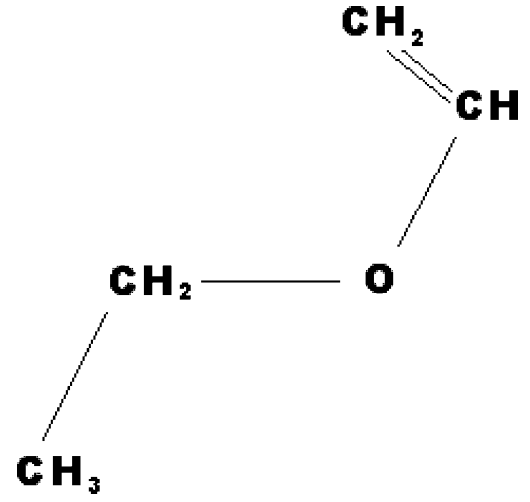

FIG. 1. Schematic of the structure of ethyl vinyl ether in its minimum-energy conformation.

\section{METHOD}

\section{A. Experimental}

The experimental apparatus has been described in previous articles, e.g., Khakoo et al. [17], so only a brief description will be given here. The electron gun and the detector employ double hemispherical energy selectors, and the apparatus is made of titanium. Cylindrical lenses are used, and the system was baked to about $130^{\circ} \mathrm{C}$ with magnetically free biaxial heaters (ARi Industries model BXX06B41-4K). The analyzer detector was a discrete dynode electron multiplier (Equipe Thermodynamique et Plasmas model AF151) with the extremely low background rate of $<0.01 \mathrm{~Hz}$ and the capability of linearly detecting more than $1 \mathrm{MHz}$ without saturating. The remnant magnetic field in the collision region is reduced to less than $1 \mathrm{mG}$ by using a double $\mu$-metal shield as well as a coil that eliminates the vertical component of the Earth's magnetic field. Typical electron currents were around 10 to $20 \mathrm{nA}$, with an energy resolution of 50 to $70 \mathrm{meV}$, full width at half maximum. The electron beam could be easily focused at $1 \mathrm{eV}$ and remained stable to within $20 \%$ over a period of several days, requiring minor tuning of the spectrometer to maintain the long-term stability of the current to within $5 \%$. The energy of the beam was established by determining the dip in the He elastic-scattering cross section due to the $2{ }^{2} \mathrm{~S} \mathrm{He}-$ resonance at $19.366 \mathrm{eV} \mathrm{[18]} \mathrm{to} \mathrm{an} \mathrm{uncertainty} \mathrm{of}$ $\pm 20 \mathrm{meV}$ during a run at a given impact energy $E_{0}$. Typically the contact potential so determined drifted by around 0.8 to $0.9 \mathrm{eV}$ over the multiweek course of the experiments. Energy-loss spectra of the elastic peak were collected at fixed $E_{0}$ values and electron scattering angles $\theta$ by repetitive, multichannel-scaling techniques. The angular resolution was $2^{\circ}$, full width at half maximum. The effusive target gas beam was formed by flowing gas through a thin aperture source $0.3 \mathrm{~mm}$ in diameter described previously [19]. This source was sooted to reduce secondary electrons and placed $6 \mathrm{~mm}$ below the axis of the electron beam, incorporated into a movable source arrangement [20]. The movable gas source method has been well tested previously in our laboratory and determines background scattering rates expediently and accurately. The vapor pressure behind the source was about 1.5 torr, and the pressure in the experimental chamber $4 \times 10^{-6}$ torr. The gas beam temperature, determined by the apparatus temperature in the collision region, was about $130^{\circ} \mathrm{C}$.
To compute momentum-transfer and integral elastic cross sections, the measured differential cross sections (DCSs) were extrapolated to $0^{\circ}$ and $180^{\circ}$. The extrapolation at forward angles used the Born-dipole form of the DCS with a dipole moment of 0.980 Debye (D) and an energy loss of $5 \mathrm{meV}$.

\section{B. Computational}

The scattering calculations were carried out using the Schwinger multichannel (SMC) method [21,22] as implemented for parallel computers [23,24]. The geometry of EVE was optimized at the level of second-order Møller-Plesset perturbation theory in the $6-31 \mathrm{G}(d)$ basis set as contained in the GAMESS electronic structure package [25], resulting in a minimum-energy configuration of $C_{s}$ point-group symmetry, with the five heavy atoms coplanar and with the vinyl and ethyl moieties, respectively, cis and trans with respect to the linking $\mathrm{O}$ atom and each other, as sketched in Fig. 1. This result agrees with the structure established by microwave spectroscopy [26]. Calculations at the same level indicate that the transtrans conformation lies 0.004 hartree (Ha) higher in energy and that the height of the intervening barrier is $0.009 \mathrm{Ha}$, as measured from the cis-trans mininum; thus at room temperature or below the cis-trans conformer should strongly predominate, consistent with the microwave data.

For the scattering calculations, the "triple zeta valence" basis set of GAMESS was used, along with a $3 d 1 s 1 p$ supplement of diffuse and polarization functions on the heavy atoms and a $2 p 1 s$ supplement on the hydrogens. Default exponents and splitting factors were used for the supplemental Gaussians. The $x^{2}+y^{2}+z^{2}$ linear combinations of Cartesian $d$ Gaussians were excluded, leaving a total of 245 one-electron functions to describe the occupied and virtual orbitals. The target electronic wave function was described at the Hartree-Fock level in this basis; the Hartree-Fock dipole moment is $1.039 \mathrm{D}$, in good agreement with the experimental value, 0.995 D [26]. For use in the SMC calculations, the virtual orbitals were transformed into modified virtual orbitals (MVOs) [27] using $a+6$ cationic Fock operator. Target polarization effects were described by allowing singlet-coupled virtual excitations into the low-energy MVOs. Specifically, singlet excitations from the 5 outermost occupied valence orbitals into the 20 lowest MVOs were coupled with all 225 virtual orbitals to form many-electron basis functions describing the target+electron system in the SMC calculation. The triplet-coupled excitation from the $\pi$ occupied orbital of the vinyl group into the corresponding $\pi^{*}$ MVO was also included to account for the expected strong influence of the low-lying $\pi \rightarrow \pi^{*}$ triplet state on the elastic channel. Finally, singlet excitations from the 10 lowest valence orbitals were also allowed, but to keep the calculations manageable, only into the 10 lowest MVOs, and coupled only to the 40 lowest MVOs to form variational basis functions. This procedure led to 13282 doublet configuration state functions (CSFs) belonging to the $A^{\prime}$ representation of $C_{s}$ and 12265 belonging to $A^{\prime \prime}$. Separate SMC calculations were carried out for $A^{\prime}$ and $A^{\prime \prime}$ and the resulting scattering amplitudes combined.

Molecules with significant dipole moments weakly scatter electrons at large impact parameters, leading to strongly forward-peaked differential cross sections (DCS). Because 
the spatially localized, square-integrable one-electron basis functions used in our SMC calculations do not fully capture such long-range scattering, we applied a correction procedure $[28,29]$ whose effect is to complement low-partial-wave scattering amplitudes taken from the SMC results, which give information about short-range scattering, with long-range scattering results computed in the first Born approximation for a point dipole. The partial-wave cutoff for the SMC amplitude was chosen to achieve a smooth match between the forward peak due to dipolar scattering and the DCS at higher angles and was increased gradually with increasing collision energy, from $\ell=3$ at $1 \mathrm{eV}$ to $\ell=10$ at 11 to $20 \mathrm{eV}$, reflecting the increasing penetration of higher angular momenta into the spatial region covered by the one-electron basis set.

\section{RESULTS AND DISCUSSION}

Our measured and calculated DCSs are shown at selected energies in Figs. 2 and 3, and the numerical values of the measured data are listed in Table I. As may be seen in the figures, the agreement between experiment and theory is good at most energies and angles. The largest deviations at low energy occur in the near-forward direction. At 15 and especially $20 \mathrm{eV}$, the calculated DCS is larger than the measured DCS at intermediate angles; as we have noted elsewhere [30], this behavior probably reflects overestimation of the intermediate-angle elastic scattering at energies where the neglected inelastic channels (ionization, in particular) have significant cross sections and thus should be treated as open and/or accounted for by an absorbing potential [31-35].

The DCS at $3 \mathrm{eV}$ does not show the clear $d \pi$ angular pattern seen in $\pi^{*}$-resonant scattering from molecules such as

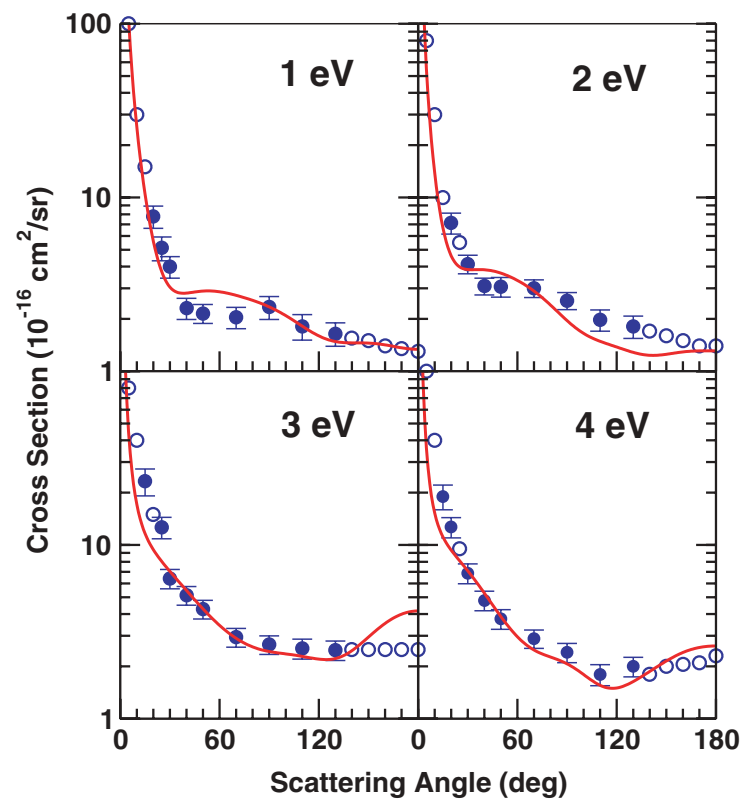

FIG. 2. (Color online) Differential cross sections for elastic electron scattering by ethyl vinyl ether. Solid blue circles with error bars are present measurements, open blue circles are extrapolated values, and the solid red curve is the present $a b$ initio calculation. The collision energy is indicated in each panel.

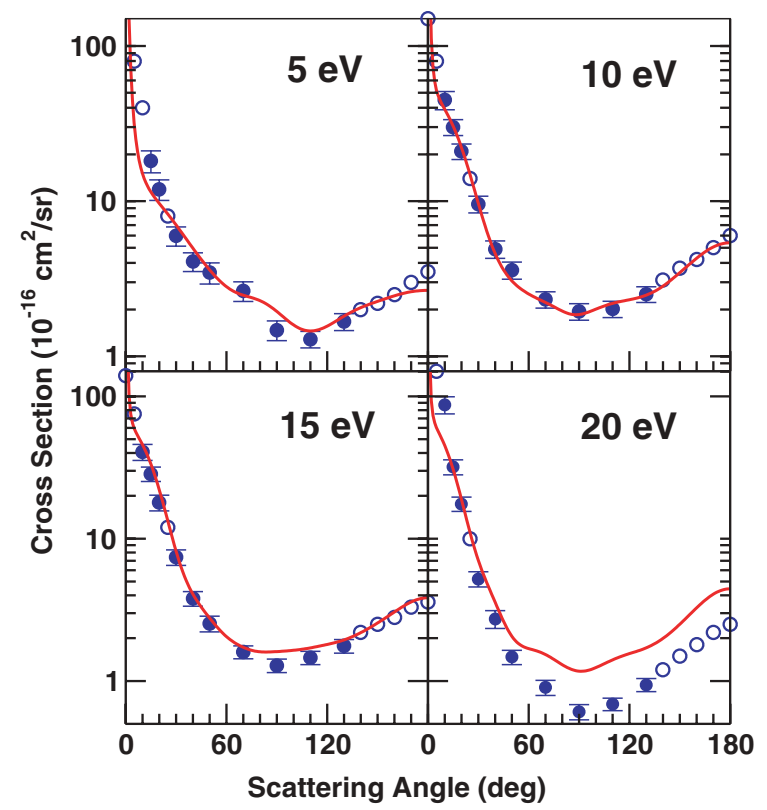

FIG. 3. (Color online) As in Fig. 2 but at higher collision energies.

$\mathrm{C}_{2} \mathrm{H}_{4}$ (e.g., Refs. [36,37]). The presence of a large nonresonant background, including dipolar scattering, may obscure the resonant scattering pattern in EVE. On the other hand, when the SMC DCS is plotted against energy for fixed scattering angles, the results (not shown) are qualitatively similar to those found for $\mathrm{C}_{2} \mathrm{H}_{4}$ (see Fig. 1 of Ref. [37]): although the resonance is visible at each angle, it is less prominent at $\sim 45^{\circ}-90^{\circ}$ than at higher or lower angles, and only at intermediate to high angles is there a pronounced minimum on the low-energy side of the peak.

Integral elastic and momentum-transfer cross sections are shown in Fig. 4. It should be noted that, at low energies, both the experimental and the calculated integral cross sections contain a significant contribution from the forward-scattering peak that is included in the Born-dipole approximation; the magnitude of this contribution can be gauged by comparing to the calculated results without the dipole correction, which are also shown in the top panel of the figure. Because of its $1-\cos \theta$ weighting, the momentum-transfer cross section is far less sensitive to dipolar scattering, and the momentumtransfer cross sections in the lower panel accordingly omit the Born-dipole correction. For both the integral and momentumtransfer cross sections the agreement between experiment and calculation is good above $2 \mathrm{eV}$, reflecting the agreement of the underlying DCSs. At 1 and $2 \mathrm{eV}$, the calculated integral cross section is significantly larger than the measured cross section. Much of this discrepancy is due to different inelasticities assumed in computing the Born-dipole forward-scattering corrections ( $1 \mathrm{meV}$ for the calculated and $5 \mathrm{meV}$ for the experimental data), both values being somewhat arbitrary approximations to a "typical" rotational energy loss; as the collision energy increases, the precise inelasticity assumed makes progressively less difference.

The top panel of Fig. 4 includes the ${ }^{2} A^{\prime}$ and ${ }^{2} A^{\prime \prime}$ symmetry components of the SMC integral elastic cross section, demonstrating that, as expected, the peak near $3 \mathrm{eV}$ arises from the 


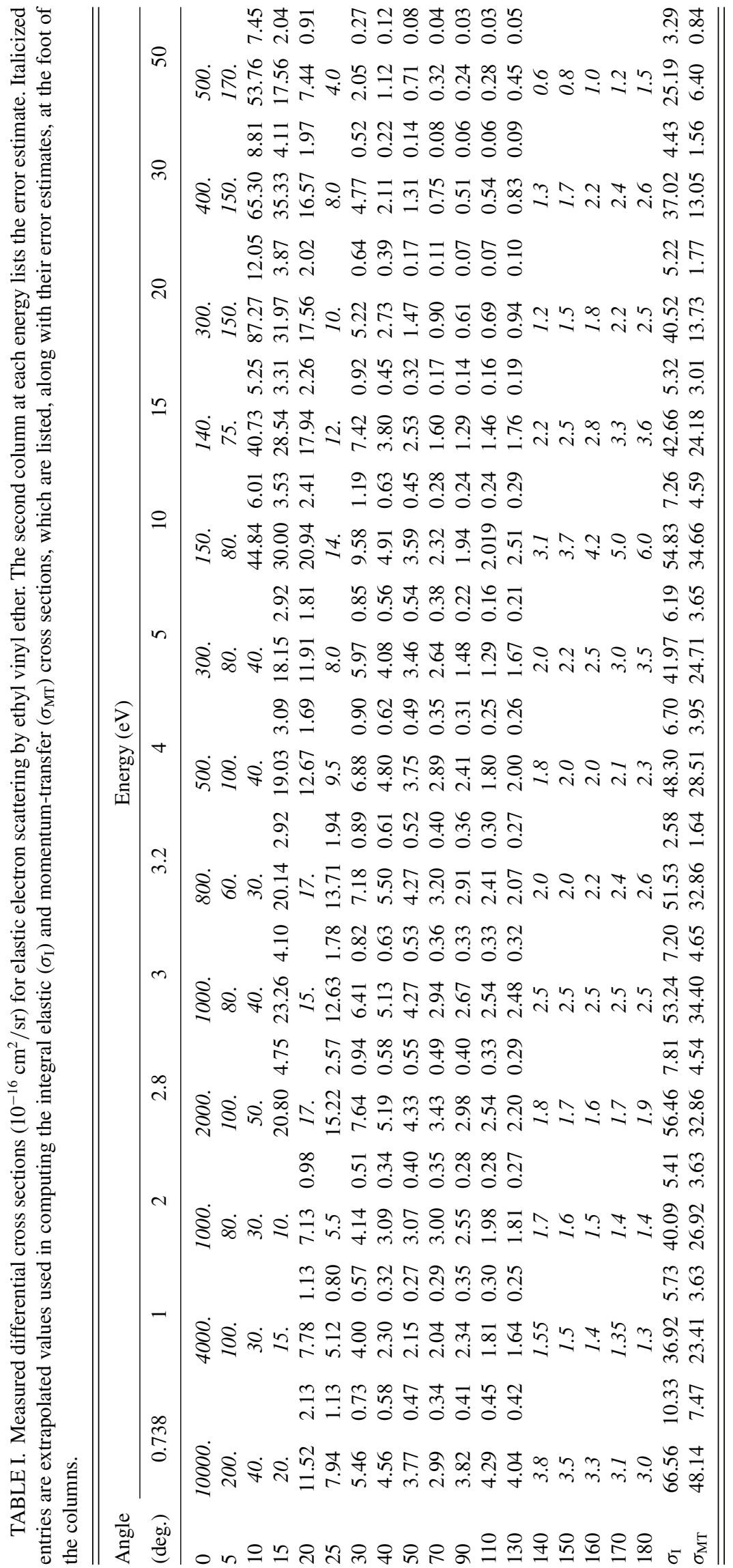




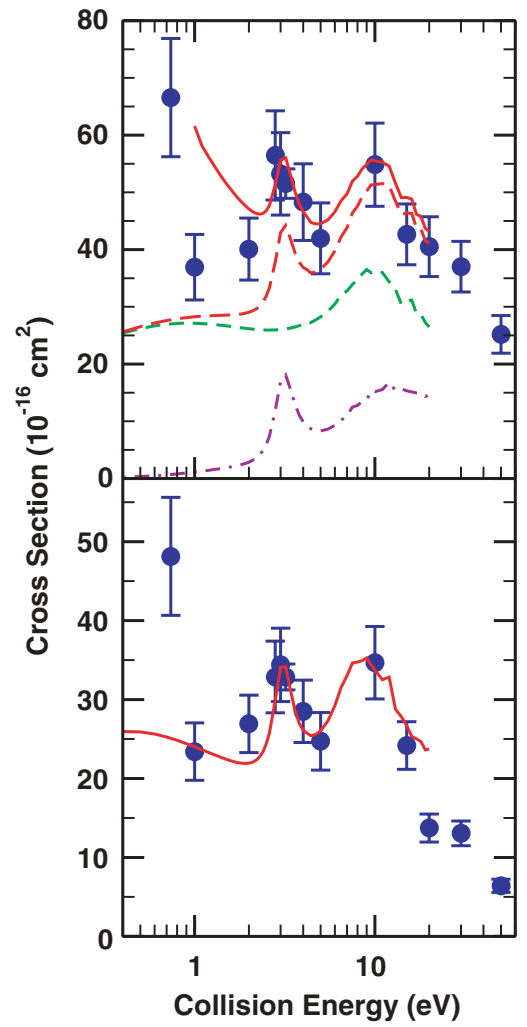

FIG. 4. (Color online) Integral elastic (top panel) and momentumtransfer (bottom panel) cross sections for electron scattering by ethyl vinyl ether. Blue circles with error bars are measured values and solid red lines are calculated results. In the upper panel, the long-dashed red line is the calculated integral cross section without the Born-dipole correction, and the short-dashed green line and dot-dashed indigo line are, respectively, its ${ }^{2} A^{\prime}$ and ${ }^{2} A^{\prime \prime}$ components. $\left(\pi \rightarrow \pi^{*}\right)^{2} A^{\prime \prime}$ shape resonance, while the broad peak near $10 \mathrm{eV}$ contains contributions from both ${ }^{2} A^{\prime}$ and ${ }^{2} A^{\prime \prime}$. The $3 \mathrm{eV}$ peak coincides with the anion-production feature observed in the DA spectra [16], supporting the assignment of that feature to the $\pi^{*}$ resonance. The broad peak near $10 \mathrm{eV}$ is likely a superposition of short-lived $\mathrm{C}-\mathrm{H}$ and $\mathrm{C}-\mathrm{C} \sigma^{*}$ resonances.

\section{CONCLUSION}

We have reported differential, integral, and momentumtransfer cross sections for low-energy electron collisions with ethyl vinyl ether. The measured and calculated cross sections generally agree well and both show evidence at $3 \mathrm{eV}$ of the presence of a $\pi^{*}$ shape resonance that has also been seen in dissociative attachment measurements [16]. In future work, we plan to clarify the role of this resonance in dissociative attachment by combining computational studies of the nucleargeometry dependence of the electron-scattering cross section with additional measurements, including measurements of the temperature dependence of the dissociative-attachment cross sections.

\section{ACKNOWLEDGMENTS}

B. Kim participated in this research under the Troy Tech research internship program. This work was sponsored by the US National Science Foundation under Grants PHY 0653452 (M.A.K.) and PHY 0653396 (V.M. and C.W.). The work of V.M. and C.W. was supported by the Chemical Sciences, Geosciences and Biosciences Divsion, Office of Basic Energy Sciences, Office of Science, US Department of Energy, and made use of the Jet Propulsion Laboratory's Supercomputing and Visualization Facility.
[1] A. M. Scheer, C. Silvernail, J. A. Belot, K. Aflatooni, G. A. Gallup, and P. D. Burrow, Chem. Phys. Lett. 411, 46 (2005).

[2] S. Denifl, P. Sulzer, F. Zappa, S. Moser, B. Kräutler, O. Echt, D. K. Bohme, T. D. Märk, and P. Scheier, Int. J. Mass Spectrom. 277, 296 (2008).

[3] D. Nandi, V. S. Prabhudesai, and E. Krishnakumar, Radiat. Chem. Phys. 75, 2151 (2006).

[4] N. Bhargava Ram, V. S. Prabhudesai, and E. Krishnakumar, J. Phys. B 42, 225203 (2009).

[5] T. N. Rescigno, C. S. Trevisan, and A. E. Orel, Phys. Rev. Lett. 96, 213201 (2006).

[6] G. A. Gallup, P. D. Burrow, and I. I. Fabrikant, Phys. Rev. A 79, 042701 (2009)

[7] T. N. Rescigno, C. S. Trevisan, and A. E. Orel, Phys. Rev. A 80, 046701 (2009).

[8] G. A. Gallup, P. D. Burrow, and I. I. Fabrikant, Phys. Rev. A 80, 046702 (2009).

[9] J. C. Y. Chen, Phys. Rev. 148, 66 (1966).

[10] T. F. O’Malley, Phys. Rev. 150, 14 (1966).

[11] J. N. Bardsley, J. Phys. B 1, 349 (1968).

[12] R. Barrios, P. Skurski, and J. Simons, J. Phys. Chem. B 106, 7991 (2002).
[13] J. Berdys, I. Anusiewicz, P. Skurski, and J. Simons, J. Phys. Chem. A 108, 2999 (2004).

[14] J. Berdys, P. Skurski, and J. Simons, J. Phys. Chem. B 108, 5800 (2004).

[15] I. Anusiewicz, J. Berdys, M. Sobczyk, P. Skurski, and J. Simons, J. Phys. Chem. A 108, 11381 (2004).

[16] C. Bulliard, M. Allan, and S. Grimme, Int. J. Mass Spectrom. 205, 43 (2001).

[17] M. A. Khakoo, C. E. Beckmann, S. Trajmar, and G. Csanak, J. Phys. B 27, 3159 (1994).

[18] J. H. Brunt, G. C. King, and F. H. Read, J. Phys. B 10, 1289 (1977).

[19] M. A. Khakoo, H. Silva, J. Muse, M. C. A. Lopes, C. Winstead, and V. McKoy, Phys. Rev. A 78, 052710 (2008).

[20] M. Hughes, K. E. James Jr., J. G. Childers, and M. A. Khakoo, Meas. Sci. Technol. 14, 841 (1994).

[21] K. Takatsuka, and V. McKoy, Phys. Rev. A 24, 2473 (1981).

[22] K. Takatsuka and V. McKoy, Phys. Rev. A 30, 1734 (1984).

[23] C. Winstead and V. McKoy, Adv. At., Mol., Opt. Phys. 36, 183 (1996).

[24] C. Winstead and V. McKoy, Comput. Phys. Commun. 128, 386 (2000). 
[25] M. W. Schmidt, K. K. Baldridge, J. A. Boatz, S. T. Elbert, M. S. Gordon, J. H. Jensen, S. Koseki, N. Matsunaga, K. A. Nguyen, S. J. Su, T. L. Windus, M. Dupuis, and J. A. Montgomery, J. Comput. Chem. 14, 1347 (1993).

[26] M. Hayashi and N. Inada, J. Mol. Spectrosc. 165, 195 (1994).

[27] C. W. Bauschlicher, J. Chem. Phys. 72, 880 (1980).

[28] T. N. Rescigno and B. I. Schneider, Phys. Rev. A 45, 2894 (1992), and references therein.

[29] M. A. Khakoo, J. Blumer, K. Keane, C. Campbell, H. Silva, M. C. A. Lopes, C. Winstead, V. McKoy, R. F. da Costa, L. G. Ferreira, M. A. P. Lima, and M. H. F. Bettega, Phys. Rev. A 77, 042705 (2008).

[30] M. A. Khakoo, J. Muse, H. Silva, M. C. A. Lopes, C. Winstead, V. McKoy, E. M. de Oliveira, R. F. da Costa, M. T. do N. Varella, M. H. F. Bettega, and M. A. P. Lima, Phys. Rev. A 78, 062714 (2008).
[31] L. M. Brescansin, P. Rawat, I. Iga, M. G. P. Homem, M.-T. Lee, and L. E. Machado, J. Phys. B 37, 471 (2004).

[32] H. Cho, R. P. McEachran, H. Tanaka, and S. J. Buckman, J. Phys. B 37, 4639 (2004).

[33] H. Cho, R. P. McEachran, S. J. Buckman, D. M. Filipović, V. Pejčev, B. P. Marinković, A. D. Stauffer, and E. J. Jung, J. Phys. B 39, 3781 (2006).

[34] H. Cho, Y. S. Park, E. A. y Castro, G. L. C. de Souza, I. Iga, L. E. Machado, L. M. Brescansin, and M.-T. Lee, J. Phys. B 41, 045203 (2008).

[35] L. M. Brescansin, L. E. Machado, M.-T. Lee, H. Cho, and Y. S. Park, J. Phys. B 41, 185201 (2008).

[36] M. Khakoo, K. Keane, C. Campbell, N. Guzman, and K. Hazlett, J. Phys. B 40, 3601 (2007).

[37] M. Allan, C. Winstead, and V. McKoy, Phys. Rev. A 77, 042715 (2008). 